\title{
Microstructure and Dynamics near an Attractive Colloidal Glass Transition
}

\author{
D. Pontoni, ${ }^{1}$ T. Narayanan, ${ }^{1, *}$ J.-M. Petit, ${ }^{1, \dagger}$ G. Grübel, ${ }^{1}$ and D. Beysens ${ }^{2}$ \\ ${ }^{1}$ European Synchrotron Radiation Facility, 38043 Grenoble, France \\ ${ }^{2}$ CEA-ESEME, ICMCB, 87, Avenue Dr. A. Schweitzer, 33608 Pessac, France
}

(Received 6 February 2003; published 5 May 2003)

\begin{abstract}
The microstructure and dynamics of a colloidal system interacting via short-ranged interparticle potential is studied by ultra-small-angle x-ray scattering and x-ray photon correlation spectroscopy. A colloidal gas-liquid type transition is induced when the short-ranged attractive interactions attain sufficient magnitude. The development of liquidlike structure is preceded by a systematic transition in the particle dynamics from diffusive to constrained motion and then completely frozen behavior. This demonstrates the existence of a jamming transition induced by strong short-ranged attractive interactions even at low packing fractions.
\end{abstract}

DOI: 10.1103/PhysRevLett.90.188301

Colloidal systems have been used extensively in the past to mimic phase transitions such as freezing, melting, and glass transition on a mesoscopic scale [1,2]. When the particles are interacting via a short-ranged attractive potential, a gas-liquid-like transition can be realized by varying the strength and the range of interaction [3]. Recently, such short-ranged attractive colloidal systems have received renewed attention [4-9]. When the strength of short-ranged attraction or stickiness becomes sufficiently greater than the thermal energy, particle motions can be jammed even at low volume fractions $(\phi)$ [5]. Prior to complete freezing of particle dynamics, the system behaves nonergodic and it is referred to as an attractive colloidal glass [5]. This is different from the conventional repulsive colloidal glass where the ergodicity is lost due to blocking of the movements of particles by the dense surrounding cages formed by their nearest neighbors [2]. Such systems usually jam under the action of an externally applied stress [10].

A short-ranged attractive interaction of controlled magnitude can be realized in several different ways. The well-known example is sterically stabilized colloidal particles in a marginal solvent [3]. Here, the hard-spherelike particles transform to sticky hard spheres at lower temperatures. A similar situation arises when a nonadsorbing polymer is added to an otherwise repulsive colloidal system [1]. In this case, the polymer induced osmotic depletion effect leads to an effective attraction between particles. There is an even more convenient way to induce a short-ranged attractive potential and thereby obtain a colloidal gas-liquid transition [11]. Certain charge stabilized colloidal systems when suspended in a binary mixture solvent near its liquid-liquid coexistence boundary, a temperature dependent absorption process can lead to a fully reversible colloidal aggregation [11]. Below the aggregation temperature $\left(T_{A}\right)$, the colloids are stabilized by repulsive electrostatic interactions. Above $T_{A}$, the adsorbed wetting layer weakens the electrostatic double layer stabilizing the suspension and result in a secondary minimum in the interparticle potential [12].
PACS numbers: 82.70.Dd, 61.10.Eq, 64.70.Pf

The transition is fully reversible and the control parameter is the temperature difference $\left|T_{A}-T\right|$.

The interparticle potential in short-ranged attractive colloidal systems can be approximately described by hard-sphere repulsion with an attractive square well [5]. The phase diagram of these systems has been extensively studied using Ornstein-Zernike integral equation in the Percus-Yevick approximation [3], and revealed the existence of binodal and spinodal regions. More recent modecoupling analysis of such systems showed several new features which include two kinetic transitions [5]. As in the case of repulsive systems, there is a critical line of glass transition in the high $\phi$ range [4,5]. A more striking feature is a second glass transition at lower $\phi$ ranges caused by strong attractive interaction [5]. The attractive and repulsive glass transition lines meet to form a reentrant region of glass-liquid-glass phases [5,9].

This Letter presents a study of microstructure and dynamics near an attractive colloidal glass transition using ultra-small-angle $\mathrm{x}$-ray scattering (USAXS) and $\mathrm{x}$-ray photon correlation spectroscopy (XPCS). The transition is induced by a temperature dependent adsorption process in a charge stabilized colloidal system. The results demonstrate that the dynamics is completely frozen as the liquidlike static structure is developed.

The experimental system consisted of Stöber silica colloids suspended in a binary mixture of 2,6 lutidine and heavy water. This system undergoes a reversible colloidal aggregation at $T_{A}$ in the one-phase region of the solvent near its bulk coexistence [11]. The details of this system and discussion about the mechanism of the aggregation process can be found elsewhere [12]. The essential features are identical for different colloid volume fractions $(\phi)$ and if heavy water is replaced by normal water except for the exact value of $T_{A}$. The mean radius of the particles deduced from the polydisperse sphere model [13] was $60.4 \mathrm{~nm}$ with a polydispersity of $8.3 \%$. Typical $\phi$ used was in the range $0.04-0.1$ and the results reported here correspond to $\phi \sim 0.08$. 
The USAXS measurements were performed at the High Brilliance beam line (ID2) at the European Synchrotron Radiation Facility (ESRF) in Grenoble, France [14]. The crossed analyzer configuration in the Bonse-Hart setup permitted to obtain intensity profiles without slit smearing and the scattered intensity, $I(q)$, over 8 orders of magnitude was acquired using a highdynamic range avalanche photodiode detector. The XPCS measurements were made at the Troika beam line (ID10A) at the ESRF [15]. The coherent part of the undulator radiation was selected by a $12 \mu \mathrm{m}$ pinhole before the sample. The homodyne intensity autocorrelation function, $g_{2}(t)$, is obtained by an ALV-5000 digital correlator. The incident X-ray wavelength $\left(\lambda_{o}\right)$ was 1 and $1.56 \AA$ for USAXS and XPCS, respectively.

Typical $I(q)$ before and after the aggregation $\left(T_{A}=\right.$ $26.805^{\circ} \mathrm{C}$ ) is shown in Fig. 1. Here, the scattering vector, $q=\left(4 \pi / \lambda_{o}\right) \sin (\theta / 2)$, with $\theta$ the scattering angle. Figure 2 depicts the corresponding structure factor, $S(q)$, obtained using the measured single particle scattering function, $P(q)$. The data clearly show the change from repulsive to attractive behavior near $T_{A}$. The short-range character of the interaction is directly evident and the low $q$ upturn in $S(q)$ typical of attractive interactions was noticed only above $26.75^{\circ} \mathrm{C}$. Even well above $T_{A}$, the height of the first maxima of $S(q)$ remained below 2.85-the Hansen-Verlet criterion for freezing [2]. Therefore, this aggregation has the features of a gas-liquid transition of colloids. The first peak position of $S(q)$ in Fig. 2(c) corresponds to particles in close contact $(q R \sim 3.24)$. In addition, the USAXS data (Fig. 1) show that the development of liquidlike structure above $T_{A}$ is associated with a significant additional scattering in the ultra-small-angle region. This excess intensity also decreases like $q^{-4}$ similar to that of $P(q)$ at large $q$ values.

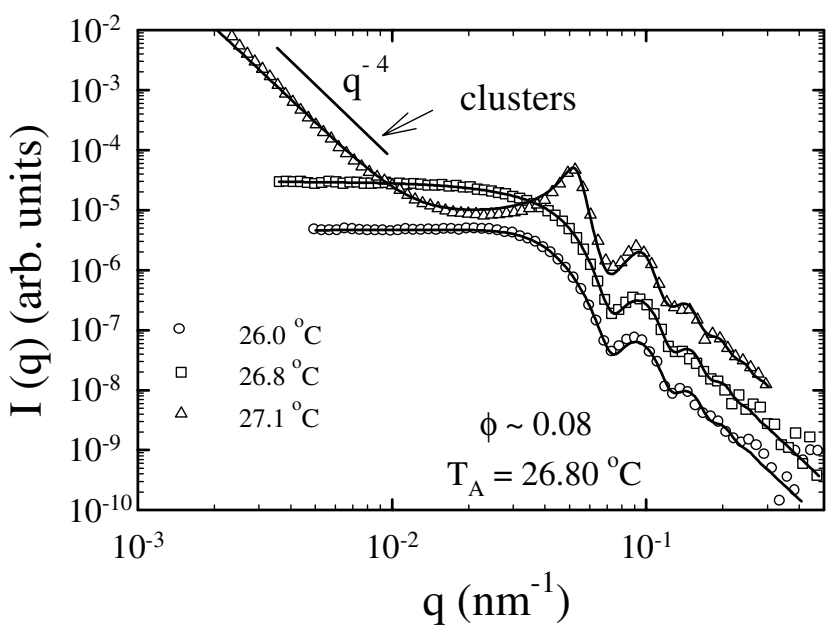

FIG. 1. Evolution of USAXS intensity in the vicinity of $T_{A}$. The continuous lines are fits using Eq. (3). The different scattering curves have been displaced for the sake of clarity.
In order to illustrate the particle dynamics across the transition, Fig. 3 depicts the behavior of $g_{2}(t)$ in the vicinity of $T_{A}$ at $q=0.019 \mathrm{~nm}^{-1}$. At this $q$ value, static $I(q)$ showed only a small variation below and just above $T_{A}$. Below $T_{A}, g_{2}(t)$ decayed exponentially with time $(t)$, $g_{2}(t)=1+g(0) \exp (-2 \Gamma t)$, where $\Gamma$ is the homodyne decay rate. This is expected for a weakly interacting system and in this $q$ range, $\Gamma=D_{C} q^{2}$, with $D_{C}$ the collective diffusion constant of the Brownian particles $[1,16]$. Near $T_{A}$, the behavior of $g_{2}(t)$ changed to stretched exponential decay, $g_{2}(t)=1+g(0) \exp \left[-(t / \tau)^{\beta}\right]$ with $1 / \tau=2 \Gamma$ when the stretching exponent, $\beta \approx 1$. This type of relaxation indicates constrained motions of particles as that near a glass transition [17,18]. Furthermore, $\beta$ value continuously decreased and within a narrow temperature range $\left(\sim 0.01^{\circ} \mathrm{C}\right)$, the system transformed to a completely frozen dynamics corresponding to time averaged $g_{2}(t)=1$ (nonfluctuating speckle) [18].

In order to deduce the parameters of interparticle pair potential, $U(r)$, above and below $T_{A}$, the measured intensities were modeled in terms of the $S(q)$ of a squarewell system obtained using the Percus-Yevick (PY) closure relation within the mean-spherical approximation $[19,20]$. For square-well attractive system, $U(r)$ has the following form:

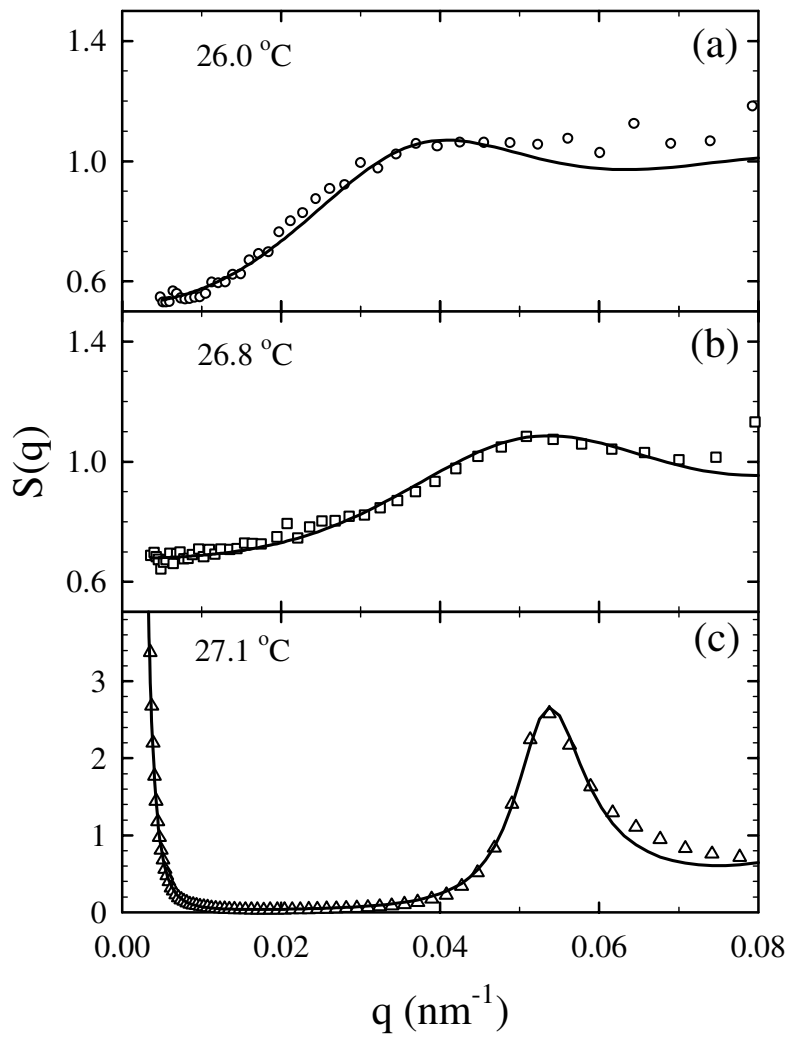

FIG. 2. Structure factor corresponding to the $I(q)$ data in Fig. 1 demonstrating the transition from repulsive (a) to attractive (b), and aggregated state (c). The slight discrepancy around $q=0.07 \mathrm{~nm}^{-1}$ arises from the $P(q)$ division. 


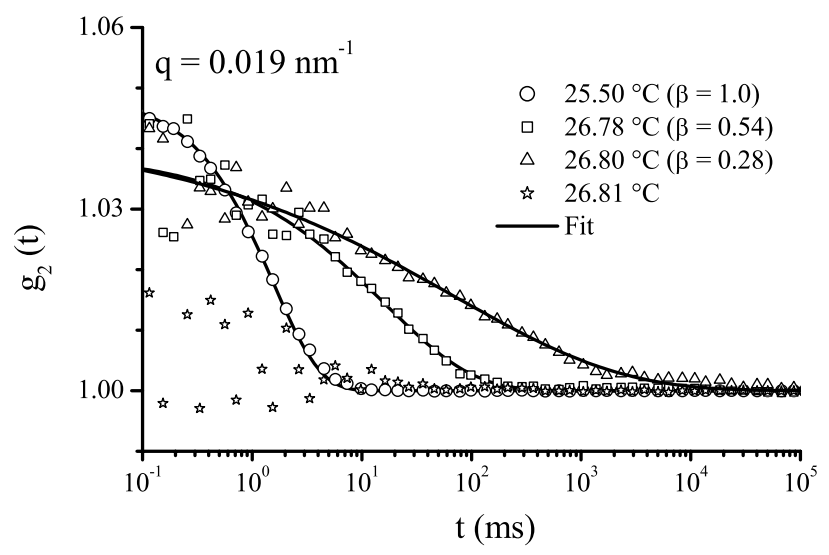

FIG. 3. Measured homodyne intensity autocorrelation function in the vicinity of $T_{A}\left(\sim 26.80{ }^{\circ} \mathrm{C}\right)$. Fitted lines correspond to stretched exponential decay with $\beta$ values indicated in the legend. Single exponential decay was observed at $25.5^{\circ} \mathrm{C}$ while $g_{2}(t)$ became flat $(\sim 1)$ at $26.81^{\circ} \mathrm{C}$.

$$
U(r)= \begin{cases}\infty & 0<r<\sigma, \\ -\varepsilon & \sigma<r<\lambda \sigma, \\ 0 & \lambda \sigma<r\end{cases}
$$

where $\sigma$ is an effective hard-sphere diameter, and $\varepsilon$ and $\lambda$ are depth and range of the square well, respectively. $S(q)$ is related to $U(r)$ through the direct correlation function, $C(r)$ [20]. $S(q)=1 /\left[1-\rho_{C} C(q)\right]$, where $C(q)$ is the Fourier transformation of $C(r)$ and $\rho_{C}$ is the colloid number density related to $\phi\left(=\pi \rho_{C} \sigma^{3} / 6\right)$. For spherical particles, $I(q)$ is related to $S(q)$ and $P(q)$ by the following simple relation [19],

$$
I(q)=N P(q) S(q),
$$

where $N \propto \rho_{C}$, and $P(q)$ is obtained from a dilute sample $(\phi \sim 0.005)$ and fitted to a polydisperse sphere scattering function [13]. Above $T_{A}$, there is a strong scattering at small $q$ 's arising from the colloidal aggregates as shown in Fig. 1. This contribution can be described by the Debye-Büche function [21] and the resulting expression for $I(q)$ is given by

$$
I(q)=N P(q)\left[S(q)+\frac{S_{M}}{\left(1+q^{2} \xi^{2}\right)^{2}}\right],
$$

where $S_{M} \propto$ average mass and $\xi \propto$ characteristic size of the clusters. The second term is significant only well above $T_{A}\left(27.05^{\circ} \mathrm{C}\right)$ where the dynamics showed completely frozen behavior. As evident in Figs. 1 and 2, the contribution arising from the two terms in Eq. (3) can be well separated.

In the fitting procedure for $I(q)$, the parameters $\sigma$ and $\rho_{C}$, were allowed to vary around their experimental values, and $\varepsilon$ and $\lambda$ were free parameters. Removed from $T_{A}$, the repulsive interaction is actually of the Yukawa form $[1,2]$ but in the case of weak interactions, $S(q)$ can be approximately described by a hard-sphere potential with an effective $\sigma(\sim 143 \mathrm{~nm})$. However, $\sigma$ approached a value close to the particle diameter $(\sim 121 \mathrm{~nm})$ near $T_{A}$. When the second term in Eq. (3) became significant, $S_{M}$ and $\xi$ were deduced independently from the low $q$ region. The continuous lines in Figs. 1 and 2 show the corresponding fits to Eq. (3). The good agreement between the data and the fits in Fig. 2 demonstrate that the square-well model captures the essence of interactions in this system. The resulting parameters at different temperatures are depicted in Fig. 4. When the system is aggregated, the effective $\phi$ within the clusters became much higher than in the bulk. This led to an apparent decrease of $\lambda$ and eventually, the $S(q)$ of interparticle interactions is adequately described by the hard-sphere part together with the Debye-Büche function. Alternatively, this implies the scenario where the particles are held together by the interfacial tension of the embedding fluid droplet [12]. The Porod-type slope observed at very small $q$ 's is consistent with dropletlike colloidal clusters [22] and the maximum value of $\xi$ was about $8 \mu \mathrm{m}$. In addition, for $\phi \sim 0.49$, PY closure tends to overpredict the structure [19] resulting in small deviations in the fit.

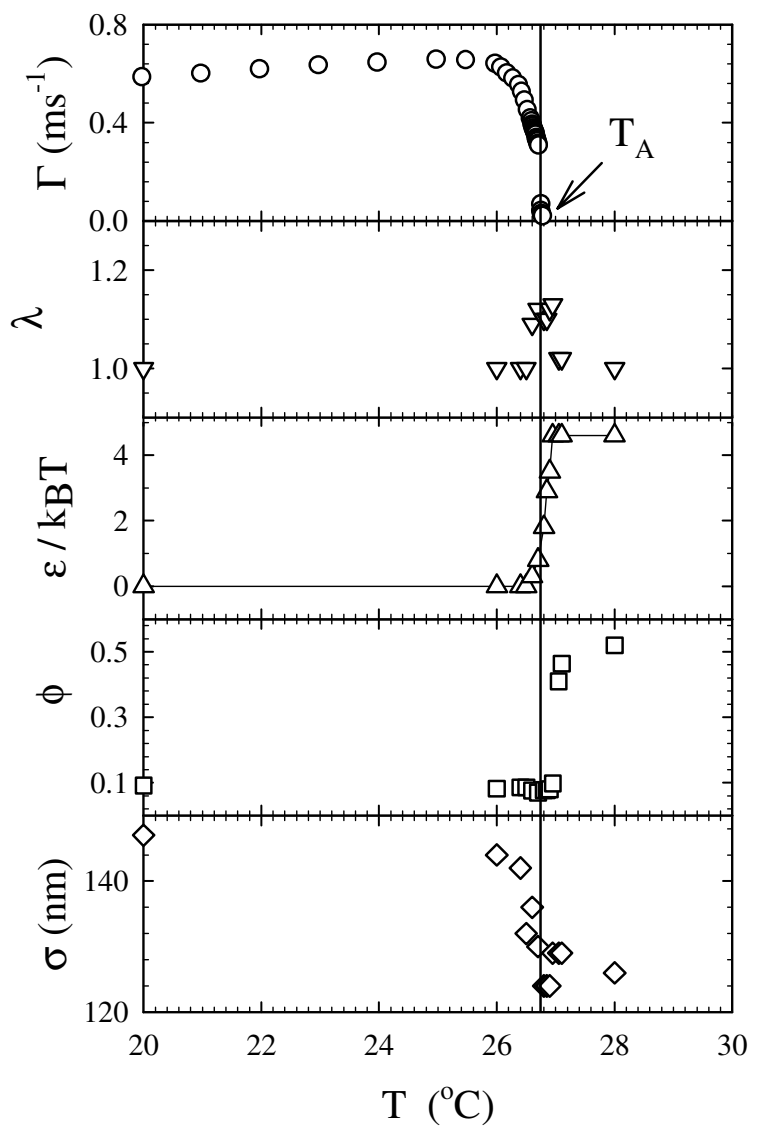

FIG. 4. Evolution of static and dynamic parameters as the system transformed from repulsive to attractive behavior. $\Gamma$ is deduced from $g_{2}(t)$ corresponding to $q=0.018 \mathrm{~nm}^{-1}$. The vertical line indicates the temperature at which $g_{2}(t)$ changed from single exponential to stretched exponential behavior. The fitted error bars are smaller than the size of the symbol.

188301-3 


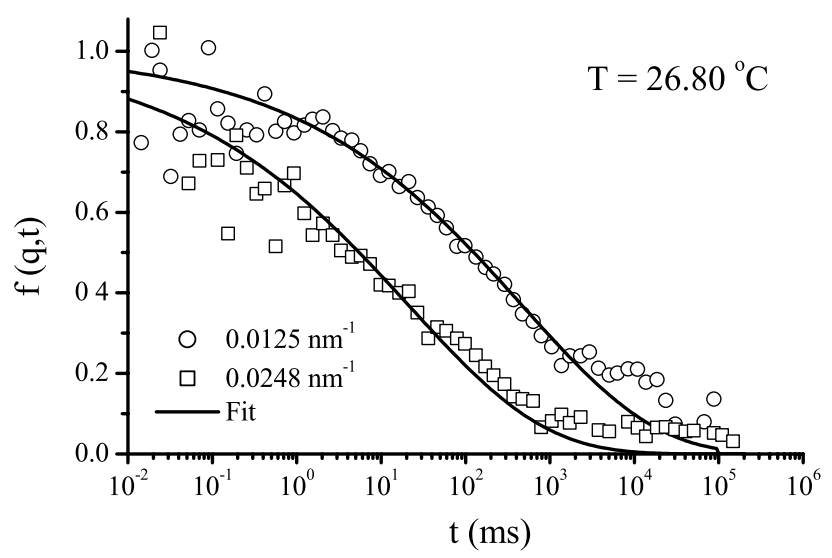

FIG. 5. Intermediate scattering functions near $T_{A}$. The continuous lines correspond to stretched exponential decay with $\beta=0.28$.

In order to make a direct comparison of static and dynamic parameters, the variation of $\Gamma$ (ergodic case) is also plotted in Fig. 4. The decrease of $\Gamma$ at a $q$ value where $S(q)$ remained constant corresponds to the slowing down of collective diffusion. The observed behavior of $\Gamma\left(=D_{C} q^{2}\right)$ near $T_{A}$ is reminiscent of a power law [23] $D_{C} \sim\left(T_{A}-T\right)^{\nu}$, with exponent $\nu \sim 0.3$. However, no universal value for the exponent $\nu$ was found among different samples studied. This suggests that $T_{A}-T$ may not be the appropriate scaling variable. The transition to stretched exponential behavior occurred when the attractive depth reached about $k_{B} T$ and the dynamics is completely frozen for $\varepsilon>2 k_{B} T$. The onset of nonergodic behavior is indicated by the intermediate scattering function, $f(q, t)$, which is given by $g_{2}(q, t)=1+$ $g(0) f(q, t)^{2}[18]$. The behavior of $f(q, t)$ near $T_{A}$ is shown in Fig. 5. The nonzero value of $f(q, t)$ at long times indicates nonergodic behavior [4,5]. A systematic study of $f(q, t \rightarrow \infty)$ is compounded by the incoherent number fluctuations resulting from the micron sized colloidal clusters traversing across the $12 \mu \mathrm{m} x$-ray beam.

In summary, the static and the dynamic behavior of colloids in the vicinity of adsorption-induced colloidal aggregation is consistent with an attractive glass transition caused by the strong short-ranged attractive interactions. A complete jamming of the particle dynamics is observed while the static structure showed liquidlike ordering. These observations are consistent with recent mode-coupling predictions concerning colloidal particles interacting via a deep square-well potential [5] and generalized jamming phase diagram of attractive colloidal systems [6]. Finally, identical transition in static behavior and nonfluctuating speckle can be observed in a variety of other colloidal systems interacting via short-ranged attractive potential.

The European Synchrotron Radiation Facility is acknowledged for the financial support and the provision of beam time. A. Moussaid, A. R. Rennie, and F. Zontone are thanked for discussion and help with XPCS measurements, respectively.

*Corresponding author.

Email address: narayan@esrf.fr

${ }^{\dagger}$ Present address: L'Oreal Research, F-92583, Clichy cedex, France.

[1] P. N. Pusey, in Liquids, Freezing and the Glass Transition, Proceedings of the Les Houches Summer School, Session LI, edited by D. Levesque, J.-P. Hansen, and J. Zinn-Justin (Elsevier, Amsterdam, 1991), p. 763.

[2] H. Löwen, Phys. Rep. 237, 249 (1994).

[3] H. Verduin and J. Dhont, J. Colloid Interface Sci. 172, 425 (1995).

[4] J. Bergenholtz and M. Fuchs, Phys. Rev. E 59, 5706 (1999).

[5] E. Zaccarelli et al., Phys. Rev. E 63, 031501 (2001); Phys. Rev. E 66, 041402 (2002), and references therein.

[6] V. Trappe et al., Nature (London) 411, 772 (2001).

[7] F. Mallamace et al., Phys. Rev. Lett. 84, 5431 (2000); M. Kapnistos et al., Phys. Rev. Lett. 85, 4072 (2000).

[8] P. N. Segre et al., Phys. Rev. Lett. 86, 6042 (2001).

[9] K. N. Pham et al., Science 296, 104 (2002); T. Eckert and E. Bartsch, Phys. Rev. Lett. 89, 125701 (2002).

[10] M. E. Cates et al., Phys. Rev. Lett. 81, 1841 (1998); A. J. Liu and S. R. Nagel, Nature (London) 396, 21 (1998).

[11] D. Beysens and T. Narayanan, J. Stat. Phys. 95, 997 (1999).

[12] B. M. Law, J.-M. Petit, and D. Beysens, Phys. Rev. E 57, 5782 (1998).

[13] M. Kotlarchyk and S.-H. Chen, J. Chem. Phys. 79, 2461 (1983).

[14] T. Narayanan, O. Diat, and P. Boesecke, Nucl. Instrum. Methods Phys. Res., Sect. A 467, 1005 (2001).

[15] G. Grübel and D. L. Abernathy, Proc. SPIE Int. Soc. Opt. Eng. 3154, 103 (1997).

[16] R. Klein, in Structure and Dynamics of Polymer and Colloidal Systems, edited by R. Borsali and R. Pecora (Kluwer Academic, Dordrecht, 2002), p. 83.

[17] A. H. Krall and D. A. Weitz, Phys. Rev. Lett. 80, 778 (1998).

[18] P. N. Pusey and W. van Megen, Physica (Amsterdam) 157A, 705 (1989).

[19] R. Klein and B. D'Aguanno, in Light Scattering: Principles and Development, edited by W. Brown (Clarendon Press, Oxford, 1996), p. 30.

[20] R.V. Sharma and K. C. Sharma, Physica (Amsterdam) 89A, 213 (1977).

[21] P.W. Schmidt, in Modern Aspects of Small-Angle Scattering, edited by H. Brumberger (Kluwer Academic Publishers, Dordrecht, 1995), p. 1; P. Debye, H. R. Anderson, Jr., and H. Brumberger, J. Appl. Phys. 28, 679 (1957).

[22] M. L. Broide, Y. Garrabos, and D. Beysens, Phys. Rev. E 47, 3768 (1993).

[23] A. Lawlor et al., Phys. Rev. Lett. 89, 245503 (2002). 